\title{
Differential interferometric phases at high spectral resolution as a sensitive physical diagnostic of circumstellar disks
}

\author{
D. M. Faes ${ }^{1,5}$, A. C. Carciofi ${ }^{1}$, Th. Rivinius ${ }^{2}$, S. Štefl ${ }^{3}$, D. Baade ${ }^{4}$, and A. Domiciano de Souza ${ }^{5}$ \\ ${ }^{1}$ Instituto de Astronomia, Geofísica e Ciências Atmosféricas, Universidade de São Paulo, Rua do Matão 1226, Cidade Universitária, \\ 05508-900 São Paulo, SP, Brazil \\ e-mail: [moser;carciofi]@usp.br \\ 2 European Organisation for Astronomical Research in the Southern Hemisphere, Casilla 19001, Santiago 19, Chile \\ 3 European Organisation for Astronomical Research in the Southern Hemisphere/ALMA, Alonso de Córdova 3107, Santiago, Chile \\ ${ }^{4}$ European Organisation for Astronomical Research in the Southern Hemisphere, Karl-Schwarzschild-Str. 2, \\ 85748 Garching bei München, Germany \\ 5 Lab. J.-L. Lagrange, UMR 7293 - Observatoire de la Côte d'Azur (OCA), Univ. de Nice-Sophia Antipolis (UNS), CNRS, Valrose, \\ 06108 Nice, France
}

Received 18 February 2013 / Accepted 30 May 2013

\begin{abstract}
Context. The circumstellar disks ejected by many rapidly rotating B stars (so-called Be stars) offer the rare opportunity of studying the structure and dynamics of gaseous disks at high spectral as well as angular resolution.

Aims. This paper explores a newly identified effect in spectro-interferometric phase that can be used for probing the inner regions of gaseous edge-on disks on a scale of a few stellar radii.

Methods. The origin of this effect (dubbed central quasi-emission phase signature, CQE-PS) lies in the velocity-dependent line absorption of photospheric radiation by the circumstellar disk. At high spectral and marginal interferometric resolution, photocenter displacements between star and isovelocity regions in the Keplerian disk reveal themselves through small interferometric phase shifts. To investigate the diagnostic potential of this effect, a series of models are presented, based on detailed radiative transfer calculations in a viscous decretion disk.

Results. Amplitude and detailed shape of the CQE-PS depend sensitively on disk density and size and on the radial distribution of the material with characteristic shapes in differential phase diagrams. In addition, useful lower limits to the angular size of the central stars can be derived even when the system is almost unresolved.

Conclusions. The full power of this diagnostic tool can be expected if it can be applied to observations over a full life-cycle of a disk from first ejection through final dispersal, over a full cycle of disk oscillations, or over a full orbital period in a binary system.
\end{abstract}

Key words. techniques: interferometric - circumstellar matter - stars: emission-line, Be

\section{Introduction}

Circumstellar (CS) disks can assume importance at various phases of the life cycles of single and binary stars. They are the place of fundamental physical processes related to properties and evolution of their central objects. Be stars are a unique class of objects that allow studying the creation, evolution and destruction of CS disks. In these main-sequence objects, the disks are fed from mass lost from their rapidly rotating central stars (Porter \& Rivinius 2003). Questions such as how the CS material can be supported against the stellar gravity and how angular momentum can be transferred from inner to external regions remained open for a long time. In the past two decades, optical and infrared interferometry played a key role in developing our understanding of these astrophysical systems. For instance, the pioneering measurements of Quirrenbach et al. (1997) provided strong evidence that the CS matter around Be stars is distributed in a geometrically thin structure (i.e., a disk). More recently, detailed measurements have demonstrated that Be disks rotate in a Keplerian fashion (Meilland et al. 2007; Kraus et al. 2012; Wheelwright et al. 2012).

The strong developments in the observations were accompanied by significant progress in our theoretical understanding of these systems. Recently, a consensus is emerging that the viscous decretion disk (VDD) model, first suggested by Lee et al. (1991) and subsequently developed by Bjorkman (1997), and Okazaki (2001), among others, is the best candidate to explain the observed properties of Be disks (Carciofi 2011; McGill et al. 2011). Detailed hydrodynamical calculations (e.g., Okazaki 2001; Jones et al. 2008) have demonstrated that VDDs rotate in a nearly Keplerian fashion, in agreement with the results from spectro-interferometry (Meilland et al. 2007; Kraus et al. 2012).

Before the spectro-interferometric era, one of the first strong indications that Be disks are Keplerian came from highresolution spectroscopy $(R>30000)$ of shell stars, which are stars with strongly rotationally broadened photospheric lines and additional narrow absorption lines. They are understood to be ordinary Be stars seen edge-on, so that the line-of-sight toward the star probes the CS equatorial disk. Hanuschik (1995) and Rivinius et al. (1999) studied the so-called central quasiemission (CQE) peaks, where the disk, under certain circumstances, causes a cusp in the deepest region of the line profile of shell stars. The existence of the CQE implies slow radial motions of the gas (much slower than the sound speed), which means that the disk is supported by rotation. 
The same mechanism that produces the CQE observed in shell-star line profiles can cause important changes in the monochromatic intensity maps of the Be star plus disk system, with observable effects on the interferometric quantities. This effect is dubbed CQE phase signature (CQE-PS; Faes et al. 2012), and here its diagnostic potential is investigated.

Similar to the spectroscopic CQEs, the CQE-PS can only be studied by high spectral resolution observations $(R>2500)$, such as can be obtained by the new generation of interferometers (AMBER/VLTI at near-infrared, Petrov et al. 2007, and VEGA/CHARA at visible range, Mourard et al. 2009).

Part of the variability observed in Be stars is associated with structural changes in their CS disks. The temporal evolution of the observables can only be put in some sort of conceptual frame with the understanding of the physical processes that control the CS dynamics. Carciofi et al. (2012) studied the $V$-band magnitude evolution of $28 \mathrm{CMa}$ during a phase of disk dissipation using the VDD model. This allowed the determination of the viscosity parameter governing the disk dynamics, as well its corresponding mass decretion rate. Haubois et al. (2012) provided another example of how CS disk and stellar parameters are intrinsically connected and how they can be inferred through a follow-up of various observables. In particular, the temporal evolution of the disk density (and corresponding continuum emission) is related to the mass-loss rate and episodes of mass injection and quiescence.

The initial studies made on the CQE profile (Hanuschik 1995; Rivinius et al. 1999) demonstrated its strong dependence on the distribution and velocity field of the CS material. Likewise, we show here that CQE-PS has a high sensitivity to structural components of the CS disk - and even to the stellar size. These parameters can then be related to the time evolution of the observables and other fundamental parameters of the system as in the cases mentioned above.

\section{Model description}

The mechanism behind the CQE-PS is the differential absorption of photospheric radiation by the CS disk (Sect. 3). This mechanism can be directly traced by the interferometric differential phase, and must be separated from other mechanisms that affect this quantity.

To study, characterize, and illustrate the CQE-PS, we adopted a realistic model for the $\mathrm{Be}+$ disk system. For the central star we adopted a rotationally deformed and gravitydarkened star whose parameters are typical of a B1 Ve star (Table 1). The stellar geometry is described by an oblate spheroid with stellar flux determined by the traditional von Zeipel effect (von Zeipel 1924), where the local effective temperature is proportional to the local surface gravity, $T_{\text {eff }} \propto g_{\text {eff }} 0.25$. For the CS disk description, we adopted the VDD model. For isothermal viscous diffusion in the steady-state regime, a state reached after a sufficiently long and stable decretion period (Haubois et al. 2012), the disk volume density has a particularly simple form $n(r)=n_{0}\left(R_{\text {eq }} / r\right)^{-m}$, where $m=3.5$ (Bjorkman \& Carciofi 2005).

The interferometric quantities depend on the spatial resolution with which the object is seen. To explore different configurations we defined the quantity $v_{\mathrm{obs}}$, the ratio between the baseline length of the interferometer, and the distance to the star. The unit used is $\mathrm{m} \mathrm{pc}^{-1}$.

The calculations presented in this paper were made with the three-dimensional, non-local radiative equilibrium code HDUST (Carciofi \& Bjorkman 2006, 2008).
Table 1. Reference Be model parameters.

\begin{tabular}{lcc}
\hline \hline Parameter & Symbol & Ref. case \\
\hline Spectral type & - & $\mathrm{B} 1 \mathrm{~V}$ \\
Mass & $M$ & $11.0 M_{\odot}$ \\
Polar radius & $R_{\text {pole }}$ & $4.9 R_{\odot}$ \\
Pole temperature & $T_{\text {pole }}$ & $27440 \mathrm{~K}$ \\
Luminosity & $L_{\star}$ & $10160 L_{\odot}$ \\
Critical velocity & $v_{\text {crit }}$ & $534.4 \mathrm{~km} \mathrm{~s}^{-1}$ \\
Rotation rate & $\Omega / \Omega_{\text {crit }}$ & 0.8 \\
Oblateness & $R_{\text {eq }} / R_{\text {pole }}$ & 1.14 \\
Gravity darkening & $T_{\text {pole }} / T_{\text {eq }}$ & 1.16 \\
\hline Disk radius & $R_{\text {disk }}$ & $10 R_{\star}$ \\
Disk-density scale & $n_{0}$ & $10^{13} \mathrm{~cm}^{-3}$ \\
Density exponent & $m$ & 3.5 \\
\hline Inclination angle & $i$ & $45^{\circ}$ \\
Spectral resolving power & $R$ & 12000 \\
Baseline/distance & $v_{\text {obs }}$ & $1 \mathrm{~m} \mathrm{pc}^{-1}$ \\
\hline
\end{tabular}

\subsection{Interferometric phases in the marginally resolved case}

The quantity registered in interferometry is the normalized complex visibility $V(\boldsymbol{u})$. It is related to the Fourier transform of the brightness distribution $I(\boldsymbol{r}, \lambda)$ of a non-coherent and extended source on the plane of sky through the Van Cittert-Zernike theorem (Born \& Wolf 1980):

$V(\boldsymbol{u})=\frac{\iint I(\boldsymbol{r}, \lambda) \exp (-2 \pi \mathrm{i} \boldsymbol{u} \cdot \boldsymbol{r}) \mathrm{d}^{2} \boldsymbol{r}}{\iint I(\boldsymbol{r}, \lambda) \mathrm{d}^{2} \boldsymbol{r}}=|V| \exp (\mathrm{i} \phi)$,

where $\lambda$ is the monochromatic wavelength and $\boldsymbol{u}$ the spatial frequency of the projected baseline $\boldsymbol{B}_{\text {proj }} / \lambda .|V|$ ranges from 1 to 0 from a fully unresolved to a fully resolved object.

A common quantity used in high spectral resolution interferometry are the differential phases $\phi_{\text {diff }}$,

$\phi_{\text {diff }}\left(\lambda, \lambda_{\mathrm{r}}\right)=\phi(\lambda)-\phi\left(\lambda_{\mathrm{r}}\right)$,

where $\lambda_{\mathrm{r}}$ is a wavelength of reference simultaneously observed. One important characteristic of differential phases arises when the target is marginally resolved, i.e., $\boldsymbol{u} \cdot \boldsymbol{r} \ll 1$. In this case, interferometric differential phases (hereafter DP or just phases) can directly map the target's photocenter (Domiciano de Souza et al. 2004, Eq. (4)):

$\phi_{\text {diff }}\left(\lambda, \lambda_{r}\right)=-2 \pi \boldsymbol{u} \cdot\left[\boldsymbol{\epsilon}(\lambda)-\boldsymbol{\epsilon}\left(\lambda_{r}\right)\right] ;$

the $\boldsymbol{\epsilon}(\lambda)$ and $\boldsymbol{\epsilon}\left(\lambda_{r}\right)$ vectors are the photocenters for $\lambda$ and $\lambda_{r}$. The usual procedure is to take $\lambda_{r}$ on the adjacent continuum of the spectral line, with $\epsilon\left(\lambda_{r}\right)=0$.

Photocenter measurements can be made at very high angular resolution using DP. One example is provided by Štefl et al. (2009), who achieved $<0.1$-mas relative photocenter astrometry in the Be star $\zeta$ Tauri.

We see below that the CQE-PS in DP occurs under quite specific circumstances, and its diagnostic potential is more relevant for some parameters than for others. To quantify this, we first studied a reference model for which the CQE-PS is not present, i.e., a model for which the canonical disk interferometric signatures apply. The adopted model parameters for the reference case are listed in the third column of Table 1.

For rotating Be disks, it is generally expected that DP have a simple S-shaped profile (Stee 1996). This can be easily understood from Fig. 1, which shows the Bry line profile, the corresponding interferometric quantities and model images at different wavelengths for the reference model. Shown are results for 
D. M. Faes et al.: Differential interferometric phases at high spectral resolution as a physical diagnostic of circumstellar disks
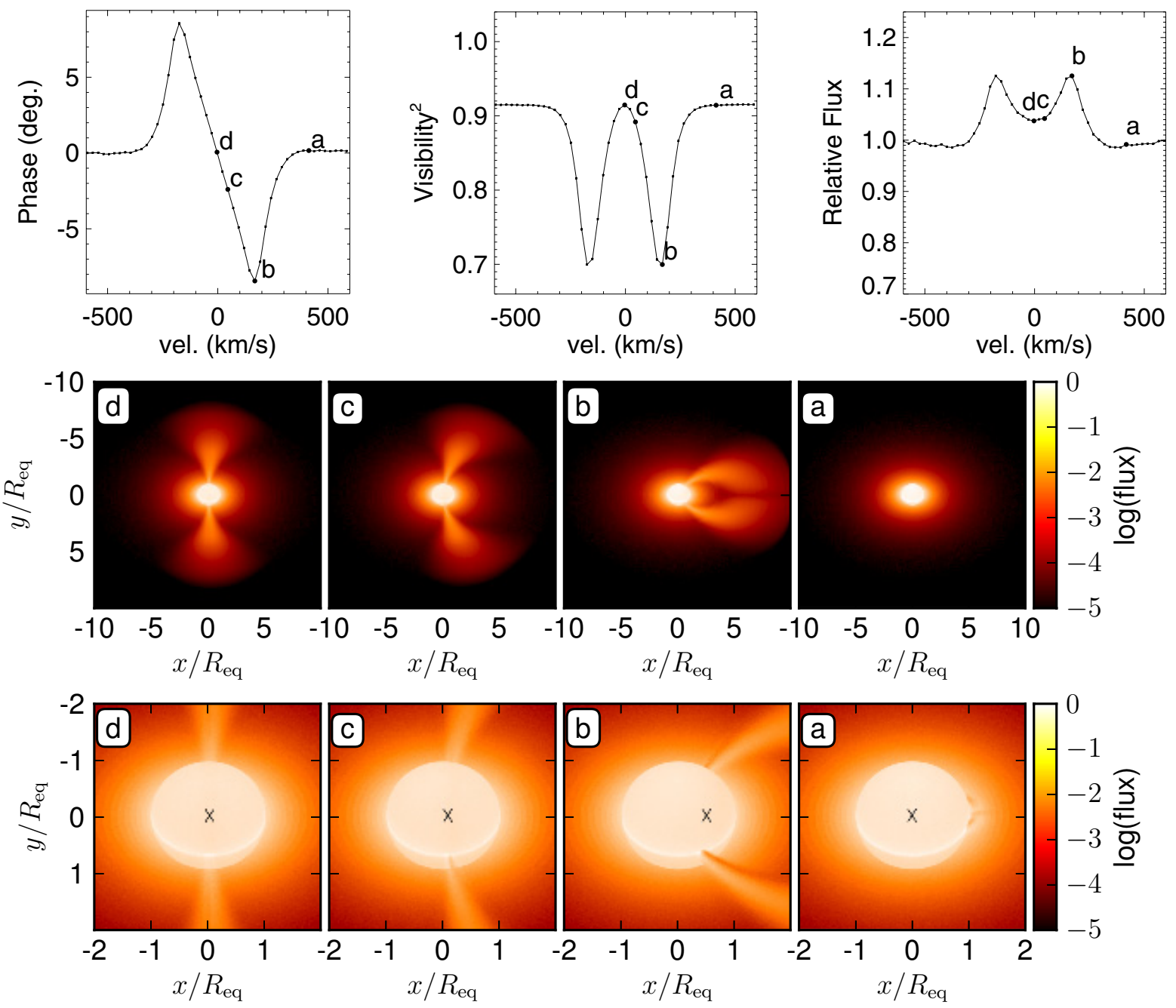

Fig. 1. Top 3 panels: interferometric Br $\gamma$ differential phases, visibilities, and line profile for the reference model. Calculations were made for $v_{\mathrm{obs}}=1 \mathrm{~m} \mathrm{pc}^{-1}$ (target marginally resolved), and baseline orientation parallel to the disk equator. Bottom panels: model images for different spectral channels (as indicated in the upper panels) at different spatial scales. The photocenter position is indicated by a black cross. The disk inclination angle is $i=45^{\circ}$. Absorption bands can be identified at velocities b) and c) but they little alter the stellar flux in the line-of-sight.

$i=45^{\circ}$. At continuum wavelengths (velocity a, in Fig. 1) the images show the star surrounded by a centro-symmetric continuum emission. We defined the DP to be zero at those wavelengths. As we move from the continuum toward line center (a to d), the line flux at a given wavelength range initially increases as a result of progressively larger emission lobes and then decreases as the emission area decreases toward line center $(\mathrm{d})$. This creates the familiar W-shaped pattern in the visibilities and the S-shape pattern in the DPs.

\subsection{Interferometric phases in the resolved case}

The association between the phase signal and the image's photocenter position is only possible if the marginally resolved condition is satisfied, since it permits the truncation of the Fourier expansion (Jankov et al. 2001). This is particularly important for Be stars because their on-sky size is wavelength dependent, mainly across emission lines whose monochromatic emission has a strong spatial dependence.

Outside the astrometric regime (i.e., $\boldsymbol{u} \cdot \boldsymbol{r} \approx 1$ ), Eq. (3) is no longer valid. A complex phase behavior is then expected since higher-order terms of the Fourier transform of the image are relevant and a direct correspondence between the DP and the

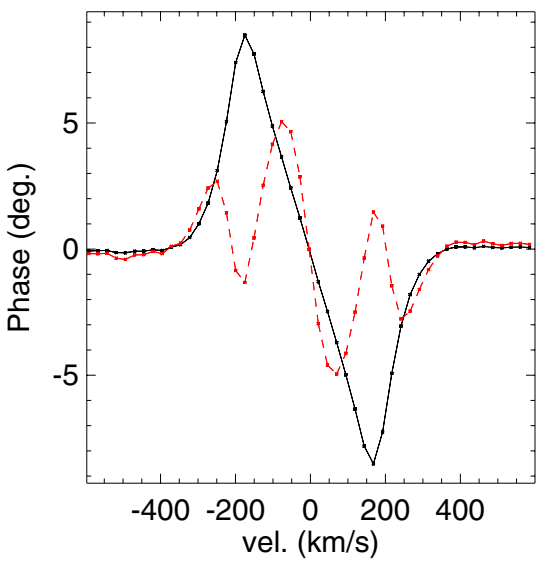

Fig. 2. Differential phase signal for the Be reference model (Sect. 2). The black (full) line corresponds to $v_{\mathrm{obs}}=1 \mathrm{~m} \mathrm{pc}^{-1}$ (astrometric regime) and the red (dashed) line to $v_{\mathrm{obs}}=2.5 \mathrm{~m} \mathrm{pc}^{-1}$ (non-astrometric regime).

photocenter position does not hold any longer. Figure 2 compares the DP signal of our reference model in the astrometric regime and outside it. The reason the curves are so different is explained in Fig. 3 that plots the interferometric quantities for 

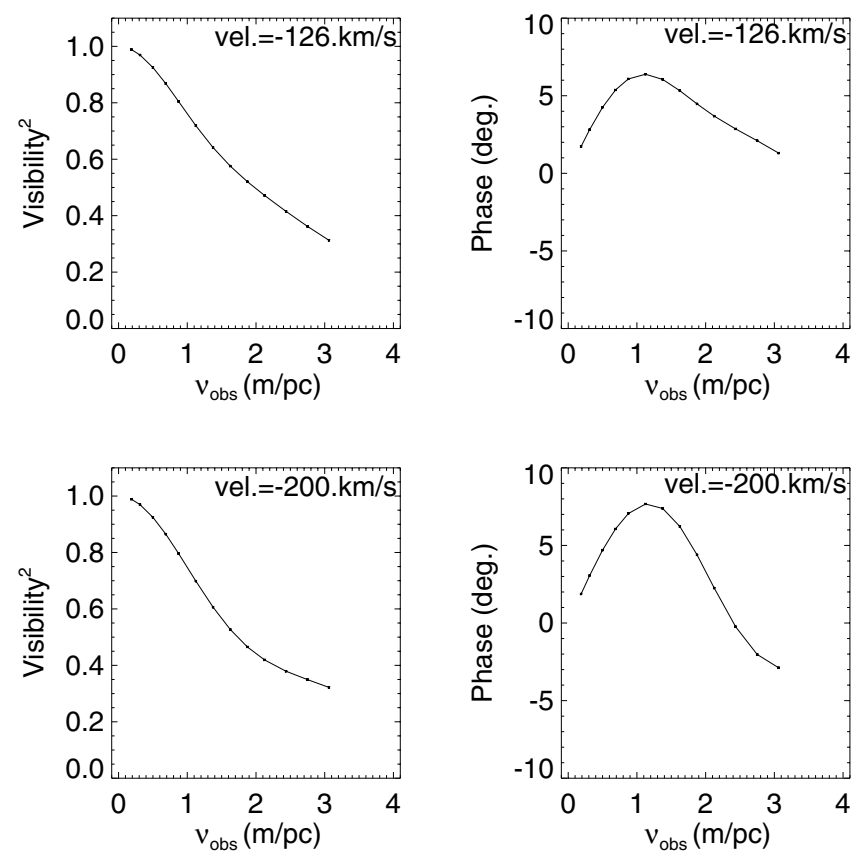

Fig. 3. Squared visibility and differential phases for the Be star reference model (Sect. 2) at radial velocities of vel. $=-126 \mathrm{~km} \mathrm{~s}^{-1}$ and vel. $=-200 \mathrm{~km} \mathrm{~s}^{-1}$ as a function of $v_{\mathrm{obs}}$, the ratio between the length of the baseline, and the distance of the target. The linear correspondence of the phase with the increasing spatial resolution $\left(v_{\mathrm{obs}} \lesssim 1.5 \mathrm{~m} \mathrm{pc}^{-1}\right)$ characterizes the astrometric regime.

two wavelengths across $\operatorname{Br} \gamma$ as a function of $v_{\mathrm{obs}}$, the ratio between $\left|\mathrm{B}_{\text {proj }}\right|$ and the target's distance $\left(v_{\mathrm{obs}} \propto \boldsymbol{u} \cdot \boldsymbol{r}\right)$. The astrometric regime corresponds to the linear growth of the phase shift with $v_{\mathrm{obs}}$ in Fig. 3. When the target becomes marginally resolved $\left(v_{\text {obs }} \gtrsim 1.5\right)$, linear correspondence no longer holds, and in the fully resolved regime the phase shift may even decrease with increasing $v_{\mathrm{obs}}$.

The interferometric signature of a Be star comprises the stellar and the disk contributions; the visibility is equally affected by both, depending on the relative flux contributions, but the phase signal is dominated by the disk emission since it comes from a large angular distance. In general, the complex phase behavior seen in Fig. 2, including change of sign, occurs always well before the disk is fully resolved. In Be stars, the presence of a small angular size star contributing a significant amount of flux moves the threshold for complex phase behavior further toward a visibility squared of unity, as seen in Fig. 3 .

Based on our simulations, the astrometric regime is approximately valid up to squared visibilities of $\approx 0.8$, which corresponds to $v_{\text {obs }} \lesssim 1.5 \mathrm{~m} \mathrm{pc}^{-1}$ for a typical Be star (we note the correspondence of $\boldsymbol{u} \cdot \boldsymbol{r}$ with $v_{\mathrm{obs}}$; the higher $v_{\mathrm{obs}}$ the higher $\boldsymbol{u}$ ). The exact value of these quantities depends on the target brightness distribution and relative flux contributions. One example of the complex behavior outside the astrometric regime is reported by Kraus et al. (2012), who modeled AMBER/VLTI data for the $\mathrm{Be} \operatorname{star} \beta \mathrm{CMi}$. In this case, a phase reversal was registered at the DP signal of the longest baseline available for this nearby target (squared visibility of $\approx 0.7, v_{\mathrm{obs}} \approx 2.2$ ).

Outside the astrometric regime a phase reversal is an example of the phenomenology that emerges entirely from longbaseline interferometry, where the phase signal no longer corresponds to displacements of the photocenter. In the text that follows all interferometric computations are provided in the marginally resolved regime (i.e., when Eq. (3) is valid) to adequately separate the CQE-PS phenomenon from the just described effects. However, the CQE-PS signal can be identified and isolated even outside the astrometric regime. The validity and characteristics of the CQE-PS under these conditions are discussed in Sect. 3.3.

\section{The CQE phase signature}

For the reference model described in Sect. 2.1, line emission from the rotating disk is the most important factor controlling the detailed shape of the DP. However, in some circumstances line absorption of photospheric light by the disk can also have a strong impact on these observables.

The spectroscopic phenomenon of the CQE was first predicted by Hanuschik (1995) for the so-called shell-absorption lines in nearly edge-on Be shell stars. The CQEs consist of narrow absorption lines well below the stellar continuum that show a central weak cusp, known as CQE peaks. Hanuschik pointed out that these peculiar profiles result from the minimum at zero radial velocity in the absorption of the photospheric flux by the rotating CS gas. However, Hanuschik expected them mainly in Fe II lines, which turned out to be formed at too large distances from the central star, where turbulence vs. orbital motion becomes strong enough to disturb the CQE signature. Later, Rivinius et al. (1999) identified these predicted CQE with previously unexplained observational features often seen in He I lines, and sometimes metallic shell lines.

This is better understood with the aid of Figs. 4 and 5, where we plot results for the reference case seen at an inclination angle $i=90^{\circ}$ (edge-on). At continuum wavelengths, the dark lane across the star is caused by continuum (free-free and bound-free) absorption in the CS disk (Fig. 4, velocity a). For the shortwavelength range corresponding to the emission line profile, the continuum opacity may be regarded as constant, so we do not expect to see changes in continuum absorption in the narrowfrequency range covered by Fig. 4. In spectral channels across the $\mathrm{Br} \gamma$ line, additional line (bound-bound) absorption of photospheric light by $\mathrm{H}$ I atoms is seen (Figs. 4, velocities b to d). This line absorption has a differential aspect because it depends on the line-of-sight velocity of the absorbing material (see below). For high line-of-sight velocities, $v_{\text {proj }}$, only a minor part of the star is affected by line absorption (Fig. 4, velocity b). Going toward lower velocities, i.e., toward the line center, this fraction increases, until it reaches a maximum at about $50 \mathrm{~km} \mathrm{~s}^{-1}$ (Fig. 4, velocity c). The precise value depends on the outer disk size and density. Closer to the line center, the lowest line-of-sight velocities are no longer absorbed (Fig. 4, velocity d), giving rise to the spectroscopic CQE effect. Spectroscopic CQEs are thus, in spite of their name, not related to any emission process, but are an absorption phenomenon.

Figure 4 can be understood with the aid of Fig. 5. We plot the projected velocity $\left(v_{\text {proj }}\right)$ in the line-of-sight vs. distance from meridian. $v_{\text {proj }}$ is expressed in terms of $v_{\text {orb }} \sin i$, the projected Keplerian orbital speed at the base of the disk $\left(v_{\text {orb }}=\right.$ $\left.\left(G M / R_{\mathrm{eq}}\right)^{1 / 2}\right)$. For a given $v_{\text {proj, }}$, the figure shows the range of distances from the meridian for which a given $v_{\text {proj }}$ occurs along the line-of-sight. The fraction steeply increases from nearly zero at the meridian, where the line-of-sight velocity is zero, to a maximum close to $0.03 v_{\text {orb }} \sin (i)$ for the reference case (lower panel of Fig. 5, vertically hatched lines). From this point on, the fraction decreases linearly with $v_{\text {proj }}$, so that at high projected velocities only light that originated close to the edge of the star can be absorbed by the disk. The dearth of absorbers with zero velocity reduces the line depth, leading to the (false) impression 
D. M. Faes et al.: Differential interferometric phases at high spectral resolution as a physical diagnostic of circumstellar disks
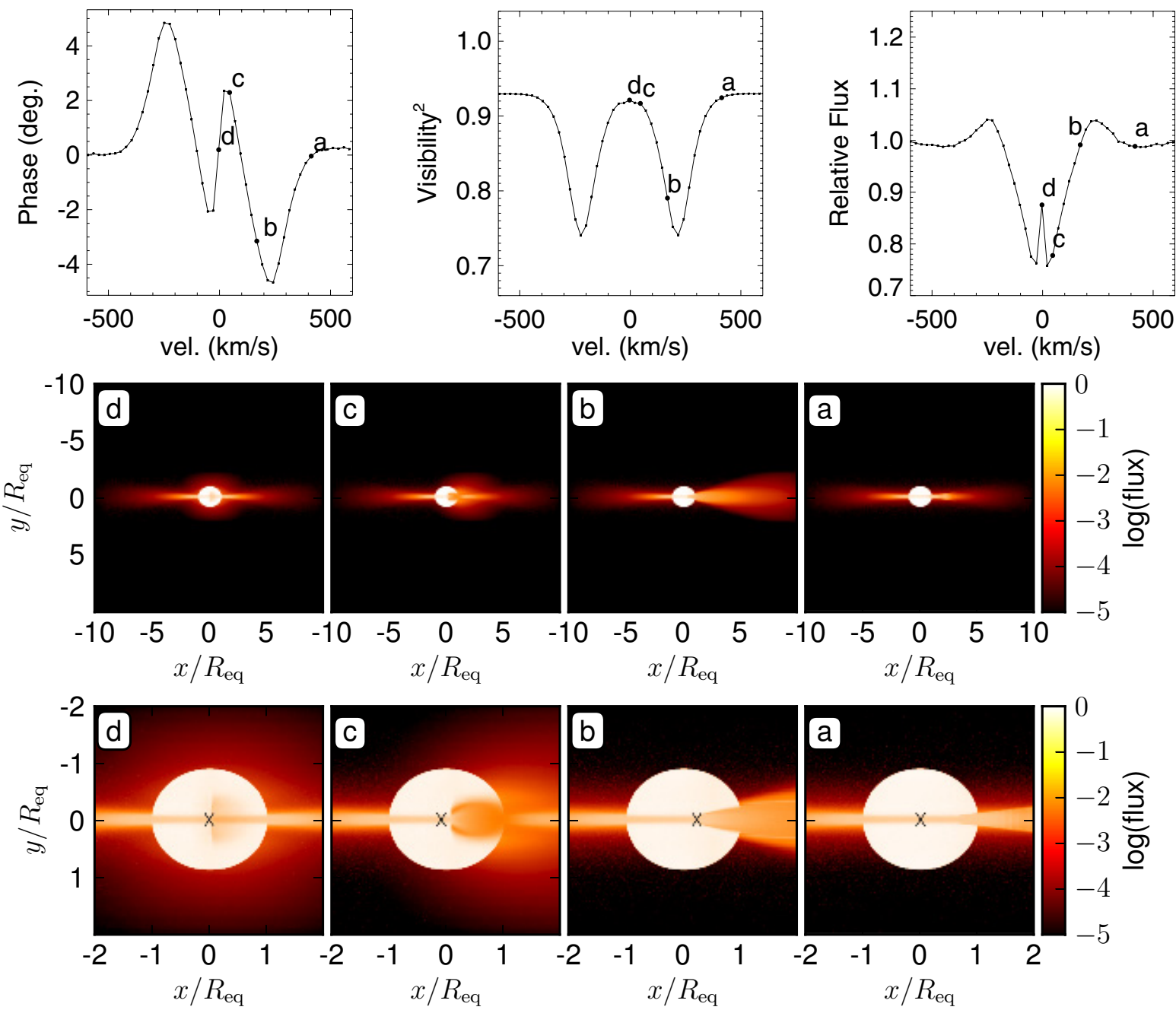

Fig. 4. Same as Fig. 1 for $i=90 \mathrm{deg}$.

of line emission. Figure 6 shows the isovelocity contours of a Keplerian disk. The figure explains why the portion of the star covered by low-velocity particles is much larger for larger disks than for smaller ones. For $R_{\text {disk }}=10 R_{\text {eq }}$, for instance, the largest surface coverage occurs for $v_{\text {proj }}=0.03 v_{\text {orb }} \sin (i)$ (lower panel of Fig. 5). The projected velocity for which this occurs increases with decreasing disk radius as $\left(R_{\text {eq }} / R_{\text {disk }}\right)^{3 / 2} v_{\text {orb }} \sin (i)$. So, for $R_{\mathrm{disk}}=2 R_{\mathrm{eq}}$ the largest surface coverage will occur for $v_{\text {proj }}=0.34 v_{\text {orb }} \sin (i)$. This shows the importance of the disk size for the spectroscopic CQE.

This line absorption across parts of the stellar disk generates a considerable decrease in the stellar flux, which in turn will affect the photocenter position of the system, with a corresponding signal in the DP. This is clearly seen in Fig. 4 as the "wiggle" in the center of the DP profile; the highest distortion is seen where the CS absorption is strongest, i.e., at about $50 \mathrm{~km} \mathrm{~s}^{-1}$. We observe the competition between spectral line emission from the disk at line-of-sight distances from the meridian higher than $1 R_{\text {eq }}$, which tends to shift the monochromatic photocenter toward one side, and disk spectral line absorption at line-of-sight distance from the meridian lower than $1 R_{\mathrm{eq}}$, which shifts the photocenter toward the opposite side. So, at wavelengths close to the line emission peak (Fig. 4, velocity b), the disk emission dominates over disk absorption and the photocenter position is shifted toward the emission lobe. For wavelengths closer to the line center (Fig. 4, velocity c), however, even though there is some disk emission, the absorption is more important and the photocenter position is shifted to the opposite direction of disk emission, with a corresponding change of sign of the DP. We conclude that the spectroscopic phenomenon of the CQE has an interferometrical counterpart (dubbed CQE-PS) that presents itself as a central reversal in the DP profile. We note that this central reversal is an intrinsic phenomenon and is independent of the reversals described in Sect. 2.2.

We expect that the interferometric CQE is more ubiquitous than its plain spectral counterpart. Figure 5 indicates that any radial motion above about $0.03 v_{\text {orb }} \sin (i)$ would destroy the spectroscopic CQE signature in a $10 R_{\text {eq }}$ disk. This is true regardless of the nature of this velocity, i.e., whether it is bulk motion (e.g., out or in-flow) or generated by turbulence. We emphasize, however, that the interferometric CQE signature is not affected, this effect relies on obscuration that travels from one side of the star $\left(-1>v_{\text {orb }} \sin (i)>-0.03\right)$ to the other $\left(0.03>v_{\text {orb }} \sin (i)>1\right)$, while the spectroscopic $\mathrm{CQE}$ requires the lowest obscuration to occur at $v_{\text {proj }}=0$.

The effects of photocenter displacement can also be traced by the interferometric closure phases. In this work we only analyze the CQE effects on DP because they strongly rely on high spectral resolution data, providing a direct measure of the target's photocenter over the projected baseline. Accordingly, all discussions here are based on the photocenter of each spectral channel which is a quantity obtained by both techniques (closure phases and DP) in an equivalent way. 

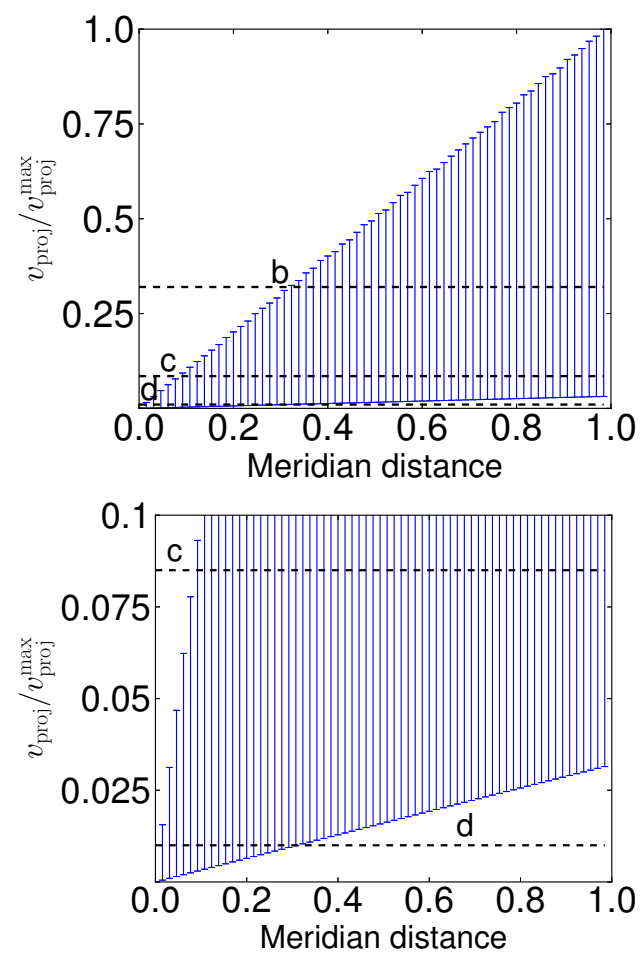

Fig. 5. Projected velocity of a Keplerian disk along the line-of-sight in units of the projected orbital speed at the base of the disk $\left(v_{\text {orb }} \sin i\right)$ vs. the normalized meridian distance (the distance to the stellar rotational axis in stellar radii). The intersection of a horizontal line with the hatched area indicates the range of meridian distances for which the projected velocity occurs. Results for different disk sizes are plotted: $R_{\text {disk }}=10 R_{\text {eq }}$, vertically hatched area; $4 R_{\text {eq }}$, horizontally hatched area; and, $2 R_{\text {eq }}$, diagonally hatched area. The velocities b to d from Figs. 1 and 4 are indicated as horizontal dashed lines. Top: full velocity range; bottom: zoom of the low-velocity components.

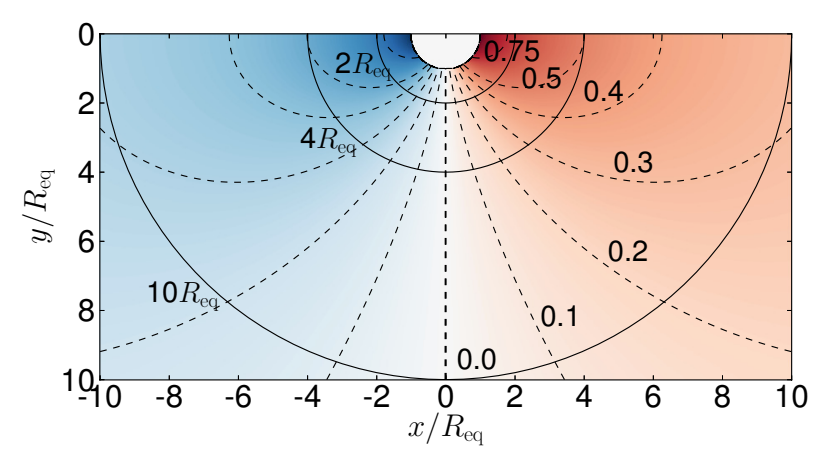

Fig. 6. Isovelocity contours (dashed lines) for a Keplerian disk. Each curve corresponds to a fraction of the projected orbital speed at the base of the disk $\left(v_{\text {orb }} \sin i\right)$, as indicated. The solid lines indicate different disk outer radii.

After understanding how disk absorption affects the DP, we now investigate under which circumstances the CQE-PS should be observable and what its diagnostic potential is for constraining the physical properties of the $\mathrm{Be}+$ disk system.

\subsection{Spectral resolution}

The CQE-PS critically depends on the spectral resolution of the interferometer, because the differential absorption that causes the CQE is only strong in a narrow wavelength range.

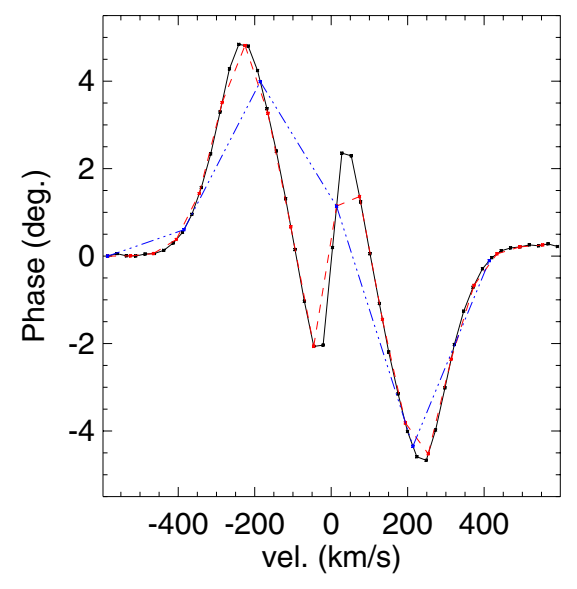

Fig. 7. Differential phase signal for the Be reference model with different spectral resolutions; $R=12000$ black (full), $R=5000$ red (dashed), and $R=1500$ blue (dot-dashed) lines. Disk inclination angle is $i=90^{\circ}$.

In Fig. 7, we see simulations of observations with different spectral resolutions for our reference model seen at $i=90^{\circ}$. The figure shows that the phase reversal would be unobservable at $R \approx 1500$, which is the medium resolution of the AMBER interferometer. Broader spectral channels also change the values of the DP since different emitting regions are sampled in each channel.

\subsection{Geometrical considerations}

Even if the physical conditions are ideal for CQE-PS, its occurrence strongly depends on the angle $i$ and less intensely on the baseline on-sky orientation.

As an absorption effect, the CQE-PS will be stronger when the system is viewed edge-on, since the densest regions of the disks are those closest to the stellar equatorial region. Clearly, when the disk is viewed face-on, this phenomenon will not occur, since there is no absorbing material in front of the star. The effects of $i$ on the CQE-PS are shown in Fig. 8, which demonstrates that the photocenter displacement due to the differential absorption is gradually weaker because less photospheric flux is absorbed for lower $i$.

Another factor that contributes to the amplitude of the CQE-PS is the baseline position angle. Because the photocenter displacements are expected to be parallel to the disk orientation, the CQE-PS will be highest when the baseline orientation is parallel to the disk major axis, as illustrated in Fig. 9.

\subsection{CQE-PS outside the astrometric regime}

The CQE-PS so far has been analyzed in the astrometric regime for which there is a direct correspondence between the target's photocenter and the DP. However, the CQE-PS is also present outside this regime, and the combination of the CQE-PS with high-resolution phase effects can result in very complex phase profiles. For instance, Fig. 10 shows how the DP signal is affected by higher spatial resolution: while the high-speed components display a complex behavior due to its more extended associated on-sky size, the CQE-PS part of the profile (low-velocity components) is still in the linear regime. Therefore, at high angular resolution the CQE-PS may even come to dominate the phase signal, which documents a phase reversal. 
D. M. Faes et al.: Differential interferometric phases at high spectral resolution as a physical diagnostic of circumstellar disks

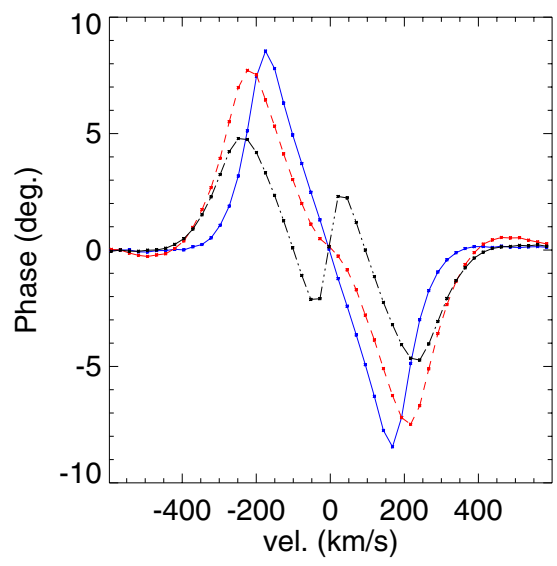

Fig. 8. Differential phase signal for the Be reference model for different disk inclinations: $i=90^{\circ}$ black (full), $i=75^{\circ}$ red (dashed), and $i=45^{\circ}$ blue (dot-dashed) lines.

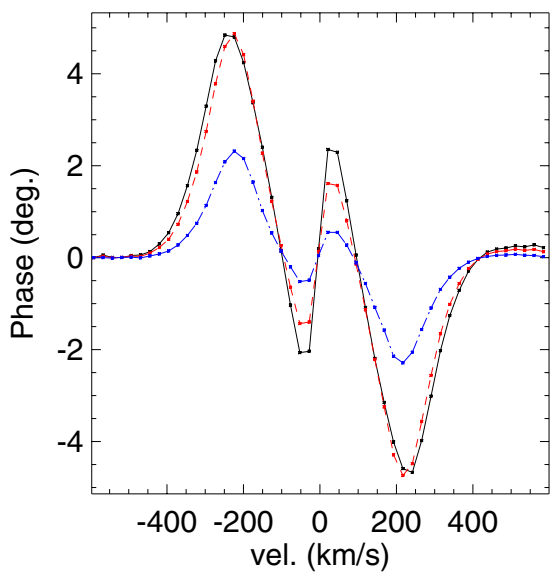

Fig. 9. Differential phase signal for the Be reference model for different angles between the projected baseline and the on-sky disk orientation: $\theta=0^{\circ}$ black (solid), $\theta=45^{\circ}$ red (dashed), and $\theta=75^{\circ}$ blue (dotdashed) lines. Disk inclination angle is $i=90^{\circ}$.

\section{Diagnostic potential}

In this section we investigate the effects of several physical parameters on the amplitude and shape of the CQE-PS. Hereafter, we assume that the interferometric baseline runs parallel to the stellar equator and $i=90^{\circ}$, since this choice maximizes the CQE-PS amplitude. All results shown were calculated for the reference model for $v=1 \mathrm{~m} \mathrm{pc}^{-1}$, which corresponds to a typical Be star of spectral type B1 V seen at a distance of $100 \mathrm{pc}$ and observed with baselines of $100 \mathrm{~m}$.

In addition to the disk size, density slope, and density scale, we also explored the effects of stellar rotation rate and spectral type, disk scale height, and turbulent speed on the CQE-PS. We found that the shape of CQE-PS is only little affected by these last parameters.

\subsection{Disk density}

The CQE-PS is a disk absorption effect, observable in the DP of Be stars together with the emission signal from the disk. The CS disk density is an important quantity for characterizing the stellar mass-loss rate and drives both the absorption and emission processes by the disk.

One can conceive two limits to the CQE-PS occurrence in terms of the disk density. Assuming a tenuous disk, absorption
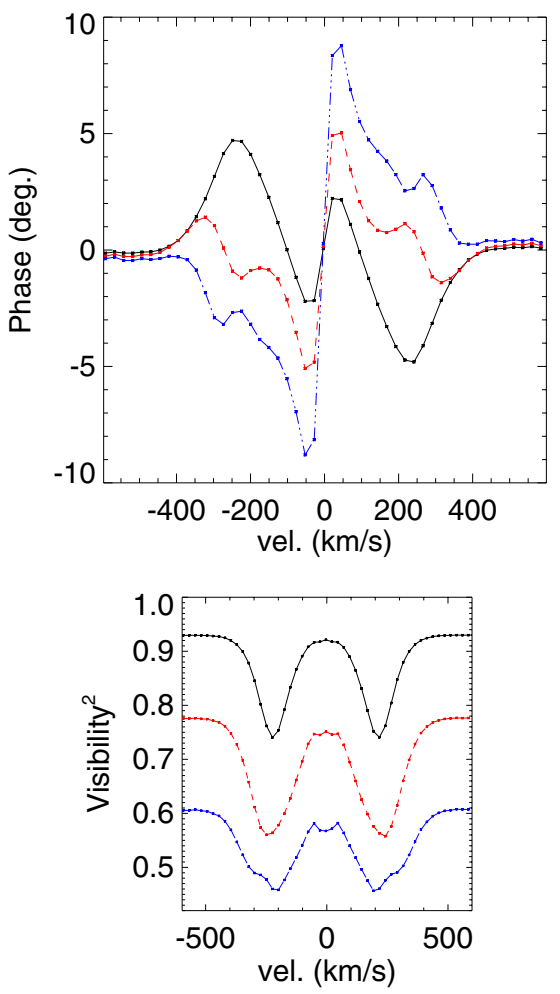

Fig. 10. Top: the differential phase signals for the Be reference model at $i=90^{\circ}$. The reference model is the black (full) line, observed with $v_{\mathrm{obs}}=1 \mathrm{~m} \mathrm{pc}^{-1}$ (astrometric regime); red (dashed) line $v_{\mathrm{obs}}=2 \mathrm{~m} \mathrm{pc}^{-1}$ and blue (dot-dashed) line $v_{\mathrm{obs}}=3 \mathrm{~m} \mathrm{pc}^{-1}$ (complex phase behavior). Bottom: the corresponding visibility signal.

(both continuum and line) probably does not block the stellar photospheric flux very much, and we expect the phase profile to be little affected by it. At the other extreme,the opacity becomes so intense for very large disk densities that absorption is high, even at continuum wavelengths. However, emission is also quite high at high densities, therefore the occurrence of the CQE-PS in the high-density regime will depend on the process (absorption vs. emission) that controls the photocenter position at the wavelengths close to the line center.

In the top panel of Fig. 11 the effects of disk density on the CQE-PS are shown in a quantitative way. We considered three values for the number density at the base of the disk $n_{0}: 10^{12} \mathrm{~cm}^{-3}, 10^{13} \mathrm{~cm}^{-3}$ (the reference model), and $10^{14} \mathrm{~cm}^{-3}$. Since the DP amplitudes of these models are very different, the DPs were rescaled in the bottom panel to better illustrate the relative effect of the differential absorption versus differential emission on the shape of the DP. The relative weight of absorption vs. emission for different densities causes considerable changes in the detailed shape of the DP, which indicates that the CQEPS can be a good quantitative diagnostic of disk density. For instance, in the high-density model (blue curve in Fig. 11) the emission almost completely outweighs the absorption, and as a result, the DP profile has just a slight hint of the CQE-PS. In our modeling we found that for a typical Be star, the density value that results in the highest contrast between the emission and absorption (i.e., the most pronounced CQE-PS) is $n_{0} \sim 10^{13} \mathrm{~cm}^{-3}$.

\subsection{Disk size}

In this subsection, we evaluate the effect of the disk outer radius on the CQE-PS. Very little is known about the actual size 

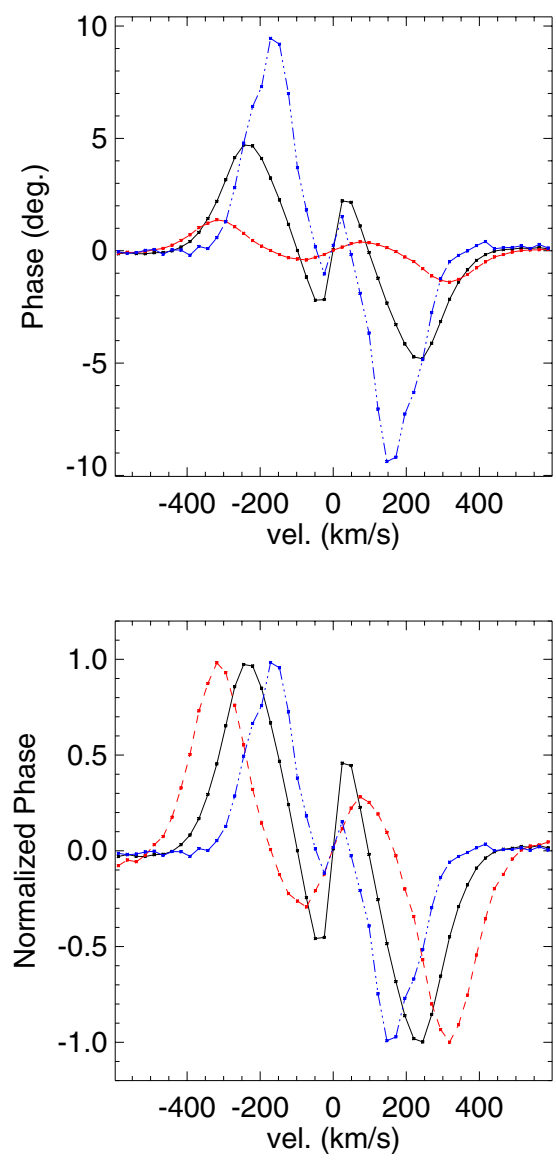

Fig. 11. Differential phase signal for the Be reference model for low$\left(10^{12} \mathrm{~cm}^{-3}\right.$, red dashed line) and high-density $\left(10^{14} \mathrm{~cm}^{-3}\right.$, blue dotdashed line) models. The reference model is the intermediate value (black full line).

of Be star disks. For known Be binaries, such as $\zeta$ Tauri (ک̌tefl et al. 2009), the disk is expected to be truncated at some radius (see, e.g., Carciofi et al. 2009), but for isolated stars there is in principle no limit for the disk size (Okazaki 2001).

Alternatively, one can define a region of the disk that is radially opaque to continuum or line radiation and therefore dominates the disk emission. The radius of this pseudophotosphere $^{1}\left(R_{\mathrm{p}}\right)$ depends on the wavelength considered; for instance, as discussed in Haubois et al. (2012), for a fully developed disk, the pseudo-photosphere extends to about $R_{\mathrm{p}}=$ $2 R_{\star}$ at the $V$ band and $R_{\mathrm{p}}=4 R_{\star}$ at the $K$ band. For line transitions such as $\mathrm{H} \mathrm{I} \mathrm{Br} \gamma$, this region can be as large as $R_{\mathrm{p}}=10-20 R_{\star}$. If the physical size of the disk is larger than the radius of the pseudo-photosphere at a given band, observations in this band constrain only the latter, and not the former. This point is illustrated in Fig. 12, where we present simulations of the reference model with different truncation radii $\left(R_{\text {disk }}=2\right.$, 4, 10 and $20 R_{\star}$ ). The models with the largest disk radii are essentially identical, which means that the size of the pseudophotosphere for the reference model is $R_{\mathrm{p}} \lesssim 10 R_{\star}$.

The situation for small disk models is quite the opposite; as shown in Fig. 12, the CQE-PS provides an unequivocal constraint to the size of the disk. Insofar as we consider smaller

\footnotetext{
1 Note that other works (e.g., Harmanec 1994, 2000) have used the term pseudo-photosphere to denote the region within which the disk viewed face-on is optically thick and so looks like an extension of the stellar photosphere.
}
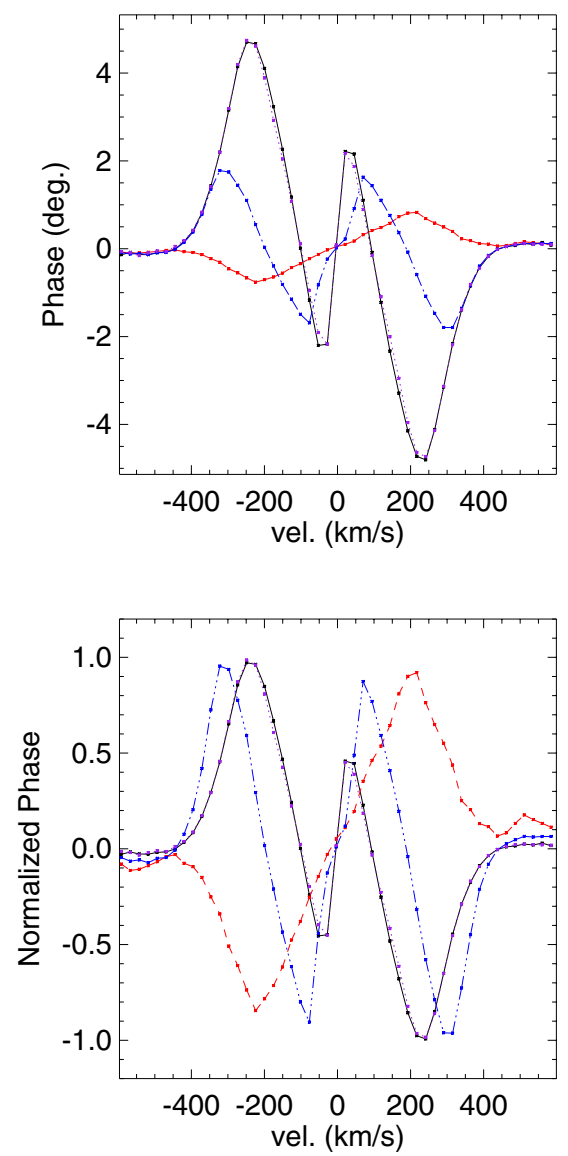

Fig. 12. Differential phase signal for the Be reference model for different disk sizes: $R_{\text {disk }}=2$ (red dashed line), 4 (blue dot-dashed line), 10 (black full line) and $20 R_{\star}$ (purple pointed line). The signals for $R_{\text {disk }}=10$ and $20 R_{\star}$ are almost coincident.

disks, the size of the emitting lobes decreases; as a result, the central reversal of the DP profile, which is a measure of disk absorption, becomes increasingly more marked (see the red curve in Fig. 12, which shows results for $R_{\text {disk }}=4 R_{\star}$ ). The net effect is that the DP peak shifts away from the line center, because the low-velocity components of the disk were removed, and the amplitude of the central reversal increases. Interestingly, for very small disks the central reversal may even dominate the entire phase profile, providing what looks like an inverted phase profile. Observations of such a disk might be misinterpreted as a disk rotating in the opposite direction! Note, however, that disk emission for such a small disk still appears as small "wings" in the DP profile where no significant absorption is present and the disk emission can be clearly seen.

\subsection{Radial density distribution}

Haubois et al. (2012) studied the temporal evolution of viscous disks around Be stars and how different mass-loss rate histories affect the disk structure. It was found that the disk density profile is highly variable in time and that is is not possible to assign one single density slope $m\left(n \propto r^{-m}\right)$ to the entire disk. However, constraining the disk slope from observations can be quite useful, since an actively forming disk will have quite different density slopes than dissipating disks. For the former case, $m$ is typically larger than 3.5, while for the latter it is $m \lessgtr 3$.

Here again the pseudo-photosphere concept can be useful. Flat disk-density distributions $(m<3.5)$ correspond to larger 

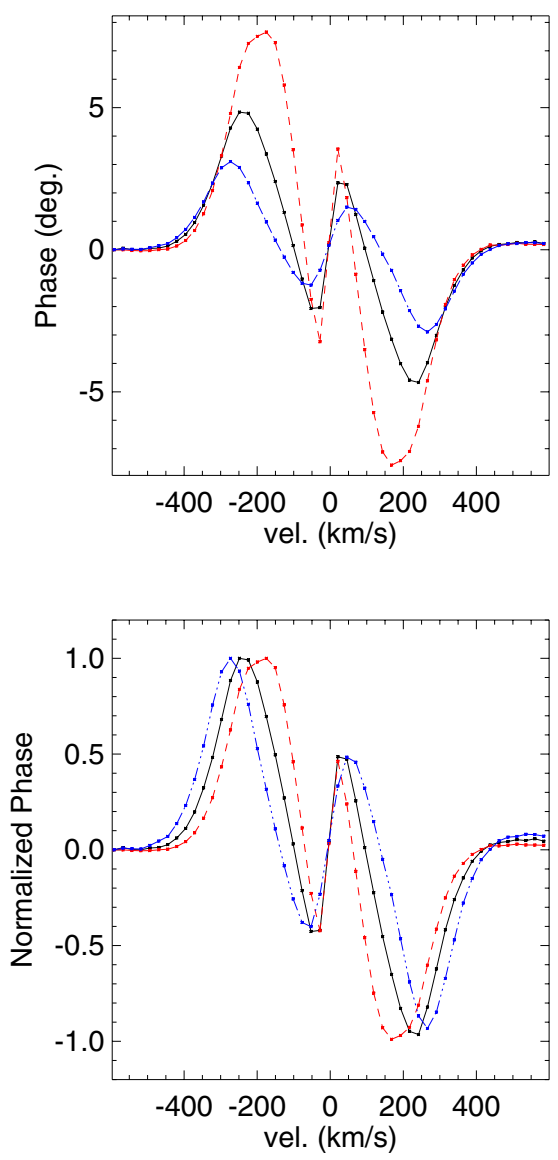

Fig. 13. Differential phase signal for the Be reference model with different radial density exponents: $m=3.0$ (red dashed line), 3.5 (black full line) and 4.0 (blue dot-dashed line).

pseudo-photospheric radii, which lead to higher contributions of lower projected velocities in the phase profile, while the converse is true for sharp disk-density profiles $(m>3.5)$. Figure 13 shows the Be reference model run for different radial density exponents. As the disk density slope decreases, the density becomes concentrated in the inner regions and the CQE-PS reversal feature broadens and increases in importance relative to the emission part of the phase profile.

Both a flat disk density distribution (low index $m$ ) and a high base density $\left(n_{0}\right)$ enhance the disk emission in a similar way, thus creating large PS amplitudes (Figs. 11 and 13). However, the CQE-PS may allow distinguishing the two cases by comparing the detailed shape of the central reversal: whereas a higher density decreases the amplitude of the phase reversal, a lower index $m$ increases it. This arises from the fact that for lowerdensity exponents the opacity decreases more slowly with radius. Therefore, a flat density slope is associated with more absorbing material in front of the star, which results in stronger contrasts between the flux from one side of the star vs. the other. This is illustrated in Fig. 5, which shows that the larger the size of the disk (or, equivalently, of the pseudo-photosphere), the larger the amount of material with the appropriate projected radial velocity.

\subsection{Asymmetric configurations and time-dependence}

The CQE-PS can be also employed to trace short-time CS variations thanks to its sensitivity to the disk inner regions. This information, linked to disk rotation, enables a disk azimuthal characterization. Similarly, it can map the evolution of the disk properties as a function of time.

The characterization of disk properties at different rotational phases would be useful for studying complex phenomena, such as the so-far unexplained triple-peaked $\mathrm{H} \alpha$ emission profiles (e.g., $\zeta$ Tauri, as in Štefl et al. 2009). This particular feature may be restricted to binary Be systems, but it is not well understood. Determining the disk configuration in such a system could offer some constraints for the influence of the companion.

Moreover, when Be stars change their brightness, this can originate in the star, the disk, or both. Although most of the observed variations are attributed to disk changes (Haubois et al. 2012), the CQE-PS can be used to settle this question because it is very sensitive to the disk part whence most of the excess flux in the visible range comes from.

\subsection{Stellar angular size}

Because CQE-PS absorption is generated across the stellar photosphere, it can be used to estimate the stellar angular size from the CQE-PS signal.

In a rotating disk, the positive (or negative) radial velocity components are projected at half of the stellar photosphere, which means that differential absorption can block at most half of the photospheric flux at a given wavelength. It can be easily demonstrated in this scenario of maximum absorption that the displacement of the photocenter due to absorption is a fraction $p \approx 2 / 5$ of the stellar radius, where $p$ is the barycenter of a unitary and uniform vertical semi-circle:

$p=\frac{2}{\pi} \int_{0}^{1} \int_{-\sqrt{1-x^{2}}}^{\sqrt{1-x^{2}}} x \mathrm{~d} y \mathrm{~d} x$.

The photocenter shift (Eq. (3)) driven by absorption can be measured as $\Delta \epsilon=p \cdot\left(R_{\mathrm{eq}} / d\right)$, where $R_{\mathrm{eq}}$ is the stellar radius, and $d$ its distance with $0<p<2 / 5$. This quantity is particularly interesting because we have an estimate of the ratio of $R_{\mathrm{eq}} / d$ in a visibility-independent measurement.

In our reference model, which can be considered representative for the stars that exhibit the phase-reversal profile, the factor $p$ is about $1 / 8$. This value, lower than $2 / 5$, is due to a smaller obscured area of the star and to the contribution of line emission by the disk. In any event, this analysis allows one to obtain a lower limit of the stellar radius.

\section{Conclusions}

With the advent of modern spectro-interferometers it became possible to measure the variation of the interferometric phase across an emission line. It was generally expected that these phase diagrams would display a simple S-shaped profile consisting of a single reversal, resulting from line emission from a rotating disk. This picture was largely confirmed by measurements made with moderate spectral resolution. However, at high spectral resolution, such as can be obtained by AMBER/VLTI, observations have shown a more complex phase profile. Conceptually, deviations from the canonical S-shaped profile can be expected in two circumstances: (i) by purely interferometric properties of observations outside the marginally resolved regime, where the differential phases no longer correspond to the photocenter of the system (i.e., end of the astrometric regime); and (ii) by physical processes accessible at high spectral resolution that affect the interferometric signal. We found for a typical Be system that the astrometric regime occurs when the visibilities are higher 
than 0.8. This corresponds to observations when $v_{\mathrm{obs}}$ is lower than $1.5 \mathrm{~m} \mathrm{pc}^{-1}$. The exact value of this quantity depends on the target's brightness distribution.

We discussed the role of radiative transfer effects (ii, above), in particular, line transfer in a rotating disk, on determining the detailed shape of the high-resolution phase signal. We demonstrated that differential line absorption by the rotating disk can cause a well-defined photocenter displacement, which in turn affects the detailed shape of the interferometric phases. We dubbed this effect central quasi-emission phase signature (CQE-PS), in reference to the fact that the same mechanism produces the CQE phase profile of shell stars (Hanuschik 1995; Rivinius et al. 1999). A detailed explanation of the CQE-PS was presented in Sect. 3.

The shape and amplitude of the CQE-PS depends on a number of parameters, both intrinsic to the system and related to the observational setup (baseline length and orientation). Using a realistic model consisting of a rotating B1 V star with a viscous decretion disk, we made an initial study to assess to diagnostic potential of CQE-PS.

Among the several model parameters explored, we found that the signal is sensitive to the CS disk density, disk size, and the radial density distribution of the CS material. Varying each of these parameters generated a characteristic shape in the differential phase diagram, which demonstrates the diagnostic potential of the CQE-PS. The amplitude of the predicted signals in phase is higher than the error for the current interferometers with high spectral resolving power (typically $\lesssim 1^{\circ}$ for AMBER/VLTI and $\$ 3^{\circ}$ for VEGA/CHARA).

Certain conditions and parameters make the effect more pronounced. Observationally, the effect is strongest for edge-on viewing (shell stars) and for baselines oriented parallel to the disk equator. Physically, the effect is strongest for base disk densities of $n_{0} \sim 10^{13} \mathrm{~cm}^{-3}$ and disks larger than about $10 R_{\star}$.

A remarkable result of the CQE-PS is its ability to make a (lower) estimate of the stellar angular size even when the observed squared visibilities are close to unity. This result may extend the distance range at which such an estimate can be made with interferometry.

The full power of this diagnostic tool will be realized when it is applied to observations over a full disk life-cycle, from first ejection through final dispersal, over a full $V / R$ cycle of disk oscillation, or over a full orbital period in a binary system. The determination of the temporal dependence of the CS parameters will enable exploring fundamental physical processes operating in the disk, which are responsible for the disk evolution timescales, the stellar mass-loss rate, and mass ejection episodes.
Acknowledgements. D.M.F., A.C.C. and A.D.S. thank the CNRS-PICS program for supporting our Brazilian-French collaboration and the present work. D.M.F. acknowledges support from FAPESP (grant 2012/04916-7). A.C.C. acknowledges support from CNPq (grant 307076/2012-1) and FAPESP (grant 2010/19029-0). This work has made use of the computing facilities of the Laboratory of Astroinformatics (IAG/USP, NAT/Unicsul), whose purchase was made possible by the Brazilian agency FAPESP (grant 2009/54006-4) and the INCT-A.

\section{References}

Bjorkman, J. E. 1997, in Stellar Atmospheres: Theory and Observations, eds. J. P. De Greve, R. Blomme, \& H. Hensberge (Berlin: Springer Verlag), Lecture Notes in Physics, 497, 239

Bjorkman, J. E., \& Carciofi, A. C. 2005, in The Nature and Evolution of Disks Around Hot Stars, eds. R. Ignace, \& K. G. Gayley, ASP Conf. Ser., 337, 75

Born, M., \& Wolf, E. 1980, Principles of Optics Electromagnetic Theory of Propagation, Interference and Diffraction of Light (London: Pergamon Press Ltd)

Carciofi, A. C. 2011, in Active OB Stars, eds. C. Neiner, G. Wade, G. Meynet, \& G. Peters, IAU Symp., 272, 325

Carciofi, A. C., \& Bjorkman, J. E. 2006, ApJ, 639, 1081

Carciofi, A. C., \& Bjorkman, J. E. 2008, ApJ, 684, 1374

Carciofi, A. C., Okazaki, A. T., Le Bouquin, J.-B., et al. 2009, A\&A, 504, 915

Carciofi, A. C., Bjorkman, J. E., Otero, S. A., et al. 2012, ApJ, 744, L15

Domiciano de Souza, A., Zorec, J., Jankov, S., et al. 2004, A\&A, 418, 781

Faes, D. M., Carciofi, A. C., Rivinius, T., et al. 2012, in Circumstellar Dynamics at High Resolution, eds. A. C. Carciofi, \& T. Rivinius, PASPC, 464, 141

Hanuschik, R. W. 1995, A\&A, 295, 423

Harmanec, P. 1994, in The Impact of Long-Term Monitoring on Variable Star Research: Astrophysics, eds. C. Sterken, \& M. de Groot, NATO ASIC Proc., 436, 55

Harmanec, P. 2000, in IAU Colloq. 175: The Be Phenomenon in Early-Type Stars, eds. M. A. Smith, H. F. Henrichs, \& J. Fabregat, ASP Conf. Ser., 214, 13

Haubois, X., Carciofi, A. C., Rivinius, T., Okazaki, A. T., \& Bjorkman, J. E. 2012, ApJ, 756, 156

Jankov, S., Vakili, F., Domiciano de Souza, Jr., A., \& Janot-Pacheco, E. 2001, A\&A, 377, 721

Jones, C. E., Sigut, T. A. A., \& Porter, J. M. 2008, MNRAS, 386, 1922

Kraus, S., Monnier, J. D., Che, X., et al. 2012, ApJ, 744, 19

Lee, U., Osaki, Y., \& Saio, H. 1991, MNRAS, 250, 432

McGill, M. A., Sigut, T. A. A., \& Jones, C. E. 2011, ApJ, 743, 111

Meilland, A., Stee, P., Vannier, M., et al. 2007, A\&A, 464, 59

Mourard, D., Clausse, J. M., Marcotto, A., et al. 2009, A\&A, 508, 1073

Okazaki, A. T. 2001, PASJ, 53, 119

Petrov, R. G., Malbet, F., Weigelt, G., et al. 2007, A\&A, 464, 1

Porter, J. M., \& Rivinius, T. 2003, PASP, 115, 1153

Quirrenbach, A., Bjorkman, K. S., Bjorkman, J. E., et al. 1997, ApJ, 479, 477

Rivinius, T., Štefl, S., \& Baade, D. 1999, A\&A, 348, 831

Stee, P. 1996, A\&A, 311, 945

Štefl, S., Rivinius, T., Carciofi, A. C., et al. 2009, A\&A, 504, 929

von Zeipel, H. 1924, MNRAS, 84, 665

Wheelwright, H. E., Bjorkman, J. E., Oudmaijer, R. D., et al. 2012, MNRAS, 423, L11 